\title{
RECENT BOOKS ON VECTOR ANALYSIS.
}

Einführung in die Vektoranalysis, mit Anwendungen auf die mathematische Physik. By R. Gans. 4th edition. Leipzig and Berlin, Teubner, 1921. 118 pp.

Elements of Vector Algebra. By L. Silberstein. New York, Longmans, Green, and Company, $1919.42 \mathrm{pp}$.

Vektoranalysis. By C. Runge. Vol. I. Die Vektoranalysis des dreidimensionalen Raumes. Leipzig, Hirzel, 1919. viii $+195 \mathrm{pp}$.

Précis de Calcul géométrique. By R. Leveugle. Preface by H. Fehr. Paris, Gauthier-Villars, 1920. lvi +400 pp.

The first of these books is for the most part a reprint of the third edition, which has been reviewed in this BuLLETIN (vol. 21 (1915), p. 360). Some small condensation of results has been accomplished by making deductions by purely vector methods. The author still clings to the rather common idea that a vector is a certain triple on a certain set of coordinate axes, so that his development is rather that of a shorthand than of a study of expressions which are not dependent upon axes.

The second book is a quite brief exposition of the bare fundamentals of vector algebra, the notation being that of Heaviside, which Silberstein has used before. It is designed primarily to satisfy the needs of those studying geometric optics. However the author goes so far as to mention a linear vector operator and the notion of dyad. The exposition is very simple and could be followed by high school students who had had trigonometry.

The third of the books mentioned is an elementary exposition of vectors from the standpoint of Grassmann. The author uses Gibbs' notation, abandoning the usual German notation of parentheses and brackets. This is a step in the right direction, though matters would be still further improved by using the Hamiltonian notation complete. The development of the tensor (dyadic)* is after Gibbs. The treatment is entirely

* Tensor as here used means dyadic, though generally it means symmetric dyadic, self-transverse matrix. 
for ordinary space, consideration of four-dimensional and higher space, useful for relativity, being deferred to the succeeding volume.

The first chapter is on vectors and plane magnitudes or quantities, and defines the various kinds of products. The second chapter is on differentiation and integration. The third is on tensors or dyadics. Simple applications are introduced where needed, the development reaching the usual elementary theorems on divergence, curl, Stokes' theorem and Green's theorem. The author uses the vector directly and only occasionally reverts to vectors as triples. There is not much attempt to elaborate formulas.

The fourth book is all that the title implies, and as a manual for working mathematicians and students is quite complete. It was written during the captivity of the author. There is a carefully worked out plan of exposition, from general notions on complex numbers to applications to electricity. In a preface of fifteen pages the general plan is explained with reasons for the eclectic notation. The sixteen chapters are analyzed, article by article, in a table of contents, making the work very accessible to the reader who wishes to consult the book. This table occupies twenty-six pages. An introduction of four pages examines the idea of quantity, and gives certain historical references of use. We shall give some idea of the scope of the work.

The first chapter is fundamental. In it the author starts with the notion of qualitative number as a given foundation, defines units, and takes as primary notion that of number considered to be the sum of numerical multiples of the units. The totality of numbers thus formed for a given set of units constitute the domain of the units. Domains are identical when every number (hypernumber) in either is also in the other. The degree of the domain is the number of linearly independent units necessary to express every number of the domain. Units are not unique, in the sense that any system of numbers of the domain which are linearly independent and in number as many as the degree of the domain, will equally define the domain. The divergence of Hamiltonian developments from Grassmannian developments comes with the idea of the product of two hypernumbers. In the former a product is always in the domain of the total set of units; in the latter a product is a hypernumber of a different grade and not in the 
domain. This very great distinction is usually omitted, overlooked, or not regarded as existing, in many treatments of vectors. It is, however, vital.

In the second chapter are defined vector, parallelogram (bivector) and spath (trivector). Addition of points and the barycentric calculus are defined, and the equations that result are deduced. The bipoint or glissant vector and the tripoint or glissant bivector are defined. The third chapter deals with combinatorial or alternating multiplication, progressive and regressive, exterior and interior. The fourth chapter applies these notions to geometry, kinematics, and statics. The notions are essentially those of Grassmann.

Chapters five and six consider the product of vectors and the scalar and vector parts of the products. These notions are essentially quaternionic. The fundamental formulas are derived from the postulated laws of multiplication of the unit vectors $i, j, k$. Chapter seven deals with the differentiation of vectors.

Chapter eight applies all the foregoing developments to geometry, kinematics, twisted curves, and curved surfaces. In each case the fundamental theorems are proved and the necessary useful formulas are deduced.

Chapter nine develops in some detail the dyadic or linear vector operator. The notation of Gibbs is introduced in part. The invariants, scalar and vector, are found, and important special cases are studied. An application is made to quadrics.

Chapters ten and eleven consider the very important nabla of Hamilton, and the various resulting differential expressions. In chapter twelve multiple integrals with the theorems of Stokes and Green, and a study of vector fields, are to be found. These three chapters lay the foundations for mathematical physics. In the remaining chapters the theory of the potential, movement of a solid, elasticity, and electromagnetism, are treated.

The book is amply supplied with examples and exercises. The typography is up to the usual excellence of the publications of the firm, and few errors occur. As a satisfactory beginning book for the various forms of vector calculus it is to be highly recommended.

James Byrnie Shaw. 